\title{
Time course of amodal completion revealed by a shape discrimination task
}

\author{
RICHARD F. MURRAY, ALLISON B. SEKULER, and PATRICK J. BENNETT \\ University of Toronto, Toronto, Ontario, Canada
}

\begin{abstract}
We measured the extent of amodal completion as a function of stimulus duration over the range of 15-210 msec, for both moving and stationary stimuli. Completion was assessed using a performancebased measure: a shape discrimination task that is easy if the stimulus is amodally completed and difficult if it is not. Specifically, participants judged whether an upright rectangle was longer horizontally or vertically, when the rectangle was unoccluded, occluded at its corners by four negative-contrastsquares, or occluded at its corners by four zero-contrast squares. In the zero-contrast condition, amodal completion did not occur because there were no occlusion cues; in the unoccluded condition, the entire figure was present. Thus, comparing performance in the negative-contrastcondition to these two extremes provided a quantitative measure of amodal completion. This measure revealed a rapid but measurable time course for amodal completion. Moving and stationary stimuli took the same amount of time to be completed $(\approx 75 \mathrm{msec})$, but moving stimuli had slightly stronger completion at long durations.
\end{abstract}

One of the challenges to object recognition is the fact that sensory information reaching the eyes is often incomplete: Objects occlude parts of neighboring objects and parts of themselves. Even though we constantly perceive partly occluded objects, we rarely notice that the visual information we receive is incomplete. Phenomenologically, we seem to complete occluded shapes immediately and effortlessly, so that we see whole, uninterrupted objects. For example, in Figure 1a, most participants report seeing a complete circle occluded by a square (Figure $1 \mathrm{~b}$ ), and not an incomplete, $3 / 4$-circle dovetailing with a square (Figure 1c). This filling in of missing sensory information is known as amodal completion (Michotte, Thinès, Costall, \& Butterworth, 1991).

Although most observers agree with such verbal descriptions of percepts of occluded objects, one could still question whether these subjective and often ambiguous impressions actually reflect important visual processes. But in fact, amodal completion greatly influences performance in many perceptual tasks, including texture segregation (He \& Nakayama, 1994), visual search (He \& Nakayama, 1992; Rensink \& Enns, 1995, 1998), primed matching (Bruno, Bertamini, \& Domini, 1997; Sekuler, 1994; Sekuler \& Palmer, 1992; Sekuler, Palmer, \& Flynn, 1994), shape matching (Gerbino \& Salmaso, 1985; Shore \& Enns, 1997), pattern discrimination (Behr-mann, Zemel, \& Mozer, 1998; Nakayama, Shimojo, \& Silverman, 1989; Ringach \& Shapley, 1996), and visual pursuit

This research was supported by NSERC Grants OGP0105494 and OGP0042133. Correspondence should be addressed to A. B. Sekuler, Department of Psychology, McMaster University, 1280 Main St. West, PC 305, Hamilton, ON, L85 4K1 Canada (e-mail: sekuler@mcmaster.ca).
(Stone, Beutter, \& Lorenceau, 2000). In these tasks, observers typically behave as if responding to the amodal completions of the stimuli, and not only to the visible, unoccluded fragments. For instance, visual search is slow if the amodal completions of the target and the distractor are similar in shape, even if the visible, unoccluded fragments are quite different (He \& Nakayama, 1992). These large and consistent behavioral effects make it possible to construct performance-based measures of amodal completion that can be used to study the perception of patterns such as that in Figure 1a without relying on subjective descriptions.

The development of such performance-based measures has made it possible to answer questions that are difficult to address using traditional introspective techniques (e.g., simply asking a participant to describe or draw what he or she sees; Kanizsa, 1979; Kanizsa \& Gerbino, 1982). For example, Sekuler and Palmer (1992) adapted the primed-matching technique (Beller, 1971; Rosch, 1975) to examine the time course of amodal completion. Their results suggested that amodal completion is not instantaneous, subjective impressions notwithstanding. Sekuler and Palmer suggested that completion actually requires a measurable amount of time (100-200 msec in their experiments), and this result has been confirmed and extended in other studies using the same primedmatching technique (Bruno et al., 1997; Guttman \& Sekuler, 2001; Sekuler, 1994; Sekuler et al., 1994). However, the primed-matching technique has its limitations. First, shape priming is difficult to detect when the stimulus duration is short, and so the technique is not ideal for mapping very rapid time courses. Second, shape priming is difficult to detect when reaction times are short, and so individual participants can only be tested in 
(a) occluded

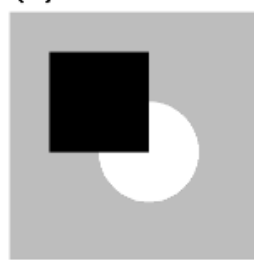

(b) whole

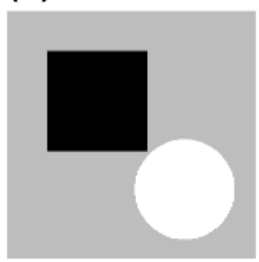

(c) clipped

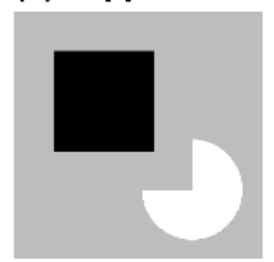

Figure 1. Amodal completion. Does (a) appear to contain the same elements as (b) or as (c)?

a few conditions of an experiment before practice makes their responses too fast to show priming. Third, the mechanisms underlying shape priming are not fully understood, and so the conclusions we can draw about amodal completion are limited by the fact that many properties of this phenomenon are simply not known. Furthermore, even in conditions under which it is appropriate to use primed matching, it is nevertheless important to demonstrate a time course for amodal completion using other methods as well, in order to be certain that the time course is not simply an artifact of one experimental method.

In this paper, we describe a new performance-based measure of amodal completion, and we use this measure to investigate the time course of completion for both moving and stationary patterns. Consider a task in which participants judge whether a rectangle is longer along its horizontal or vertical axis, in three conditions (Figure 2). ${ }^{1}$ In the complete condition, the complete rectangle appears alone. In the occluded condition, the corners of the rectangle are hidden behind four negative-contrast squares (i.e., darker than the background). In the fragmented condition, the corners are hidden behind four zero-contrast squares (i.e., having the same luminance as the background). In the fragmented condition, the same parts of the rectangle are visible as in the occluded condition, but the visible line segments do not form T-junctions with adjacent surfaces and so do not appear to be pieces of a single partially occluded rectangle (Helmholtz, 1910/1962; Kellman \& Shipley, 1991; Ratoosh, 1949).

How would one expect performance on this shape discrimination task to vary across the three conditions? Observers are highly sensitive to small deviations from perfect symmetry (Regan \& Hamstra, 1992), so one would expect performance to be very good in the complete condition. One would expect performance to be worse in the fragmented condition, perhaps simply because much of the stimulus has been deleted or because attention must be spread across several objects (i.e., line segments) rather than being confined to a single object (Baylis \& Driver, 1993; Behrmann et al., 1998; Duncan, 1984). The critical question for our experiments is, How well do participants perform in the occluded condition, relative to the other two conditions? The only difference between the occluded and fragmented conditions is the presence or absence of four occluders that provide no information as to the correct response. If occlusion cues confer no benefit, then performance should be identical in the two conditions. On the other hand, if the occluders induce amodal completion, then the occluded rectangle will be completed into a whole rectangle, and performance may be as good as in the complete condition.

Thus, the level of performance in the occluded condition, relative to the fragmented and complete conditions, can be taken as an index of amodal completion. When performance in the occluded condition is better than in the fragmented condition, we attribute this to amodal completion having been induced by the occluders. When performance in the occluded condition is no better than in the fragmented condition, we take this to mean that amodal completion has not occurred. In the following experiments, we examined the effect of stimulus duration on performance in these three conditions, to determine the time course of amodal completion for moving and stationary stimuli.

\section{EXPERIMENT 1}

\section{Method}

Participants. One author (R.F.M.) and 3 University of Toronto students participated in the experiment. Two participants were psychophysically experienced and aware of the hypotheses being tested, and 2 were inexperienced and naive. All participants in both experiments in this paper reported normal or corrected-to-normal vision.

Stimuli. There were three visibility conditions: complete, occluded, and fragmented (Figure 2). In the complete condition, the stimulus was an outlined white rectangle $\left(70 \mathrm{~cd} / \mathrm{m}^{2}\right)$ on a gray background $\left(20 \mathrm{~cd} / \mathrm{m}^{2}\right)$. The thickness of the rectangle's outline was $0.10^{\circ}$ of visual angle. The height and width of the rectangle varied across trials, as explained in the Procedure section, but, on average, were approximately $2.0^{\circ}$. In the occluded condition, the stimulus was the same as in the complete condition, except that four dark gray squares $\left(15 \mathrm{~cd} / \mathrm{m}^{2}\right)$ occluded the corners of the rectangle. The occluders measured $2.6^{\circ}$ on each side and were positioned symmetrically about the center of the display, such that $0.80^{\circ}$ of each edge of the rectangle was unoccluded. In the fragmented condition, the stimulus was the same as in the occluded condition, except that the four occluders had the same luminance as the background $\left(20 \mathrm{~cd} / \mathrm{m}^{2}\right)$, rendering them invisible. In all conditions, a small white fixation dot appeared at the center of the display.

The three visibility conditions were crossed with two motion conditions: moving and stationary. In the moving condition, the rectangle moved through a circular arc, its center tracing a circle

\section{(a) complete}

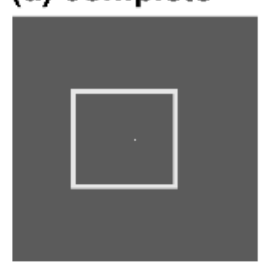

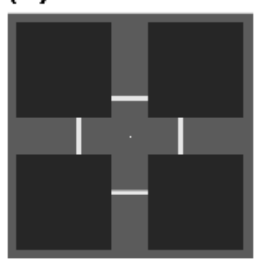

(b) occluded

\section{(c) fragmented}

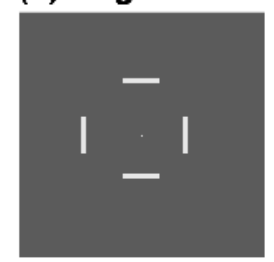

Figure 2. Stimuli in Experiment 1. 
of radius $0.20^{\circ}$ about the center of the display. The starting position of the rectangle on this circular orbit was chosen randomly on each trial. The period of the orbit was $500 \mathrm{msec}$, and the rectangle's orientation remained constant throughout. Only the rectangle moved: In the occluded and fragmented conditions, the four dark gray or zero-contrast occluders were stationary. In the stationary condition, the rectangle was placed at a different random point on the circular orbit on each trial but did not move from this point during the trial.

The six visibility $\times$ motion conditions were crossed with six duration conditions, in which the stimulus durations were 15, 30, 45, 60,105 , or $210 \mathrm{msec}^{2}$ The video refresh rate was $67 \mathrm{~Hz}$, so these stimulus durations corresponded to $1,2,3,4,7$, and 14 video frames, respectively. At the one-frame duration, the moving and stationary conditions were identical, because the stimulus could not move within a single frame.

Each trial consisted of a fixation interval $(1,000 \mathrm{msec})$, followed by the stimulus $(15-210 \mathrm{msec})$, followed by a mask of high-contrast white noise (power spectral density $=5.6 \times 10^{-4} \mathrm{deg}^{2}$ ) that remained on the screen until the participant responded. In the occluded condition, the occluders were shown during the fixation and response intervals as well as during the stimulus interval, to strengthen the impression of a rectangle appearing behind four occluders.

Stimuli were displayed on an AppleColor 13-in. monitor $(640 \times$ 480 resolution; pixel size $=0.397 \mathrm{~mm}$, frame rate $=67 \mathrm{~Hz}){ }^{3}$ The participants viewed the stimuli binocularly from a distance of $114 \mathrm{~cm}$. Head position was stabilized with a chin-and-forehead rest.

Procedure. Each participant participated in ten 1-h sessions, on separate days. The first 5 sessions showed stimuli from the moving condition, and the last 5 showed stimuli from the stationary condition. A single session comprised 18 blocks of 100 trials, one block for each of the 18 visibility $\times$ duration conditions. The 18 blocks were presented in a different random order in each session. On each trial, the participants viewed a complete, occluded, or fragmented rectangle (depending on the block's visibility condition) and pressed one of two response keys to indicate whether the rectangle appeared to be longer horizontally or vertically. Auditory feedback indicated whether the participant's response was correct. Within each block, the rectangle's aspect ratio (i.e., height/width ratio) varied from trial to trial according to two interleaved staircases, converging on $71 \%$ and $79 \%$ correct performance and ranging over 15 aspect ratios logarithmically spaced between 1.01 and 1.30. On each trial, an aspect ratio was chosen from a staircase, and it was decided randomly whether the stimulus would be longer horizontally or vertically; on horizontal trials, the reciprocal of the chosen aspect ratio was used. The absolute size of the rectangle was randomized across trials by randomly setting the length of the shorter side to between $1.8^{\circ}$ and $2.2^{\circ}$, so that neither the height nor the width alone could be used to perform the task.

The best-fitting (maximum-likelihood criterion) Weibull psychometric function was computed for each block of trials, and discrimination threshold was defined as the aspect ratio corresponding to $75 \%$ correct responses. Five thresholds were obtained for each of the 36 visibility $\times$ motion $\times$ duration conditions, for each participant.

\section{Results and Discussion}

Moving condition. Figure 3 a shows the results of the moving condition for a typical participant, plotting the complete, occluded, and fragmented thresholds as a function of stimulus duration. At shorter durations, occluded thresholds were almost as high as fragmented thresholds. At longer durations, occluded thresholds were much lower, typically one half to one third of the fragmented thresholds, and in most cases were not significantly different from thresholds in the complete con-

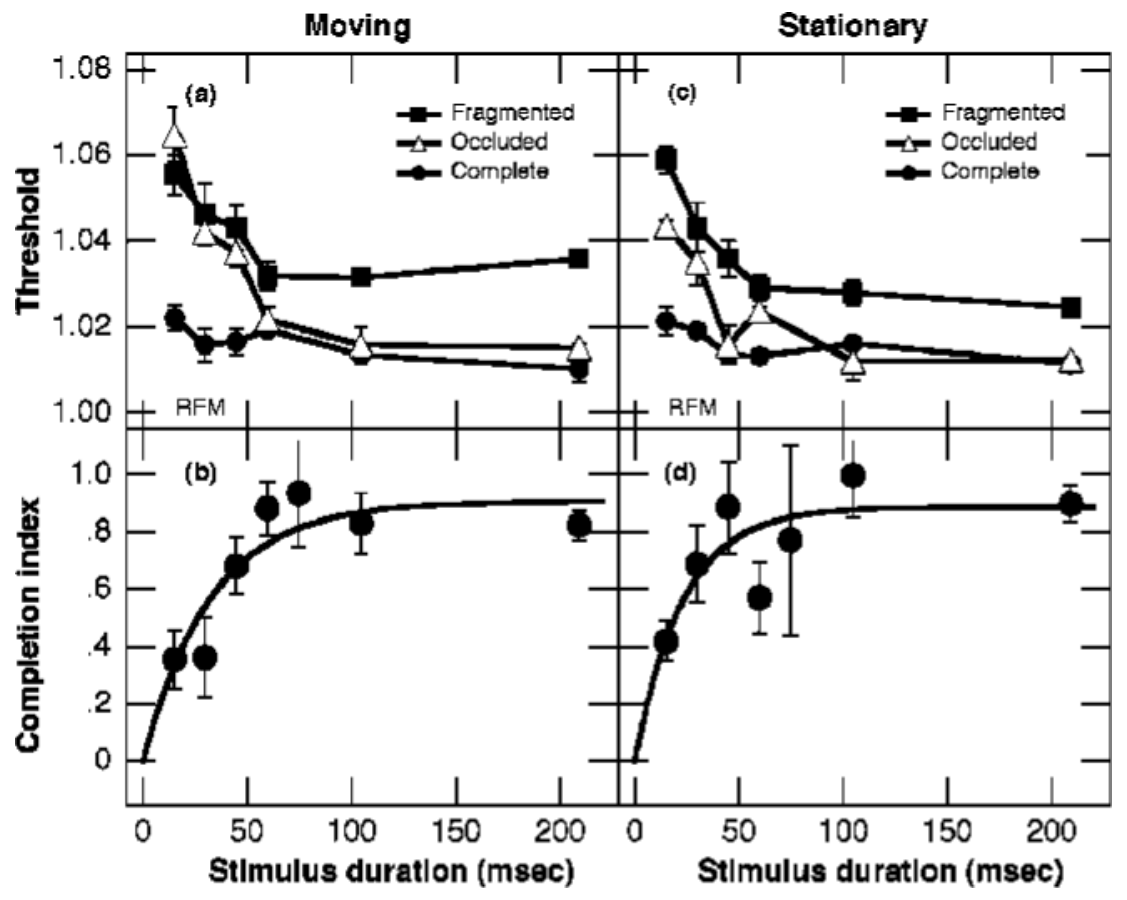

Figure 3. Results of Experiment 1. The left column shows results from the moving condition, and the right column shows results from the stationary condition. The top row shows aspect ratio thresholds for a single ty pical participant, and the bottom row shows completion indices averaged across participants. Error bars indicate 1 standard error. 
dition. For this participant, the occluded and complete thresholds converged at $60 \mathrm{msec}$, suggesting that amodal completion required approximately $60 \mathrm{msec}$ to occur.

To quantify the extent of amodal completion at each duration, we defined a completion index $c$ to be calculated from each participant's mean complete, occluded, anid fragmented thresholds:

$$
c=\frac{\text { fragmented }- \text { occluded }}{\text { fragmented }- \text { complete }} .
$$

Recall that the only difference between the occluded and fragmented stimuli was that the occluded stimulus contained dark squares that provided occlusion cues but that gave no information as to the correct response. If the occluded threshold was the same as the fragmented threshold, indicating that occlusion cues did not make the task easier, then $c=0$. If the occluded threshold was the same as the complete threshold, indicating that the participants performed as well with the partially hidden rectangle as with the complete rectangle when occlusion cues were present, then $c=1$. Averaged across participants, $c$ was near 0 at shorter durations and rose to near 1 at longer durations (Figure $3 \mathrm{~b}$ ). We fitted the increase in $c$, averaged across participants, with an exponential decay from 0 to $\gamma$, with the time constant $\tau$ and longduration asymptote $\gamma$ as free parameters:

$$
c(t)=\gamma\left(1-e^{-t / \tau}\right) .
$$

The maximum-likelihood fit of this function to the time course of $c$ gave an estimate of $\tau$ equal to $33 \mathrm{msec}$, with a $95 \%$ confidence interval of $21-44 \mathrm{msec}$, and an estimate of $\gamma$ equal to 0.91 , with a $95 \%$ confidence interval of 0.81-1.01. However, in Equation 2, $c$ is only $0.63 \gamma$ when $t=\tau$, so $\tau$ itself is not a good indicator of the time required for full completion. In this article, we define the time required for effective completion to be $2 \tau$, at which point $c$ equals $0.86 \gamma$. In the moving condition of this experiment, $2 \tau$ equaled $66 \mathrm{msec}$.

The advantage of occluded stimuli over fragmented stimuli at longer durations shows that the participants benefited from occlusion cues that induce amodal completion. However, the advantage disappeared at shorter durations, indicating that completion took approximately $66 \mathrm{msec}$. Thus, although completion does take time, it occurs rapidly. In fact, one participant had a completion index of $c \approx 1$ at even the shortest duration.

Conceptually, amodal completion is closely related to visual grouping, because it is one way in which the visual system treats disconnected regions of a stimulus as belonging together. Motion, in the guise of "common fate," is an important cue for some kinds of grouping. For example, motion may be particularly important in helping the visual system group together relevant contours for amodal completion (Kellman \& Spelke, 1983; Shore \& Enns, 1997). If motion is an important cue for amodal completion, then it is hardly surprising that completion should weaken at shorter durations, where motion signals are weaker (Snowden \& Braddick, 1989;
Watamaniuk, 1993; Watamaniuk, Sekuler, \& Williams, 1989) and, in the limit of a one-frame stimulus duration, are eliminated entirely. This possibility was addressed by the stationary condition, which we discuss next.

Stationary condition. Figure $3 \mathrm{c}$ shows the results of the stationary condition for a typical participant. Just as in the moving condition, occluded thresholds were near fragmented thresholds at shorter durations and were near complete thresholds at longer durations. (Again, one participant showed full completion $[c \approx 1]$ at all stimulus durations; this was the same participant who showed this result in the moving condition.) Averaged across participants, the completion index $c$ grew with a time constant of $\tau=23 \mathrm{msec}$ (95\% confidence interval $=$ $8-38 \mathrm{msec}$ ) to an asymptote of $\gamma=0.89$ ( $95 \%$ confidence interval $=0.80-0.98)($ Figure $3 \mathrm{~d})$, so effective completion required $2 \tau=46 \mathrm{msec}$.

The fact that the same general pattern of results emerged with and without motion shows that motion is not essential for improved performance in the occluded condition. In particular, completion was not stronger at long durations than at short durations in the moving condition solely because motion signals were stronger.

These results can be interpreted in ways that do not depend on visual completion at all. In the occluded condition, the participants could have judged the positions of the edge fragments relative to the occluding squares, whereas in the fragmented condition, no such landmarks were available. It is unclear why position cues would be useful only at long durations, or why they would give a completion index of approximately $c=1$ at long durations, but this is nevertheless an important difference between the occluded condition and the others. Furthermore, in this experiment, the occluders had much lower Weber contrast than the rectangle (occluders $=-0.25$, rectangle $=+2.5$ ), so the occluders may have been backwardmasked by the postmask, causing a rise in occluded thresholds at short durations. This explanation is not entirely plausible, because the occluders were always present, whereas the rectangle was flashed only briefly, so the total spatiotemporal contrast energy of the occluders was actually much higher than that of the rectangle. Nevertheless, the results would be more convincing if the occluders and the rectangle were equally susceptible to disruption by the postmask. In Experiment 2, we controlled for position cues across conditions and equated the contrast magnitudes of the rectangles and the occluders.

Finally, in Experiment 1, the participants completed the moving condition before the stationary condition, so possible practice effects make it difficult to compare the moving and stationary time courses. In Experiment 2, we interleaved moving and stationary blocks.

\section{EXPERIMENT 2}

\section{Method}

Participants. Seven new participants from the University of Toronto participated. All were psychophysically experienced but unaware of the hypotheses being tested. One of these participants 
(a) complete

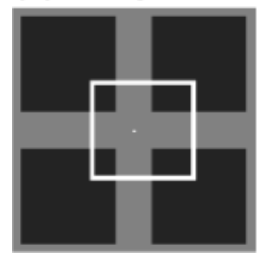

(c) fragmented

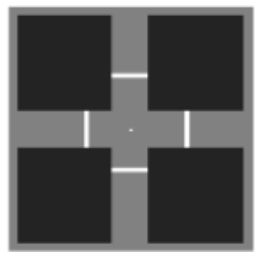

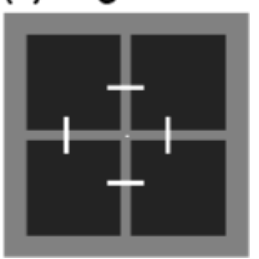

Figure 4. Stimuli in Experiment 2.

was unable to perform the task in the occluded and fragmented conditions at the critical, shortest duration, so his data were excluded from the analyses.

Stimuli. The stimuli were similar to those in Experiment 1, the main difference being that the four dark squares in the occluded stimulus were present in all three visibility conditions (Figure 4). In the complete stimulus, the four dark squares were themselves occluded by the rectangle. The layout of the occluded stimulus was the same as in Experiment 1. In the fragmented stimulus, the four squares were shifted inward toward the center of the display by $0.40^{\circ}$, eliminating the T-junctions as occlusion cues and preventing the visible line segments from appearing to be pieces of a partially occluded rectangle. Because the four dark squares were present in every condition, landmarks were always available for relative position judgments.

The luminances of the background and the stimuli also differed from the values used in Experiment 1: The background luminance was $35 \mathrm{~cd} / \mathrm{m}^{2}$, the luminance of the rectangle was $56 \mathrm{~cd} / \mathrm{m}^{2}$, and the luminance of the dark squares was $14 \mathrm{~cd} / \mathrm{m}^{2}$. This gave the rectangle a Weber contrast of 0.60 and gave the dark squares a Weber contrast of -0.60 , so that all parts of the stimulus were approximately equally susceptible to disruption by the mask. Because of constraints on the monitor's luminance range, the mask's power spectral density was reduced to $1.3 \times 10^{-4} \mathrm{deg}^{2}$ in this experiment. Finally, the stimulus durations used in Experiment 2 were 15, 30, 60,120 , and $210 \mathrm{msec}$, differing slightly from those used in Experiment 1 .

Procedure. The procedure was the same as in Experiment 1, except that the participants participated in only 4-6 sessions, rather than 10. Half the sessions showed stimuli from the moving condition, and half showed stimuli from the stationary condition; the two

types of sessions were interleaved.

\section{Results and Discussion}

Figure 5 shows the results of the moving and stationary conditions, plotting the completion index $c$, averaged across participants, as a function of stimulus duration. As in Experiment 1, occluded thresholds were near fragmented thresholds at short durations, resulting in $c \approx 0$, and were near complete thresholds at long durations, resulting in $c \approx 1$. In the moving condition, the completion index grew with a time constant of $\tau=57 \mathrm{msec}(95 \%$ confidence interval $=38-77 \mathrm{msec}$ ) to an asymptote of $\gamma=1.00$ (95\% confidence interval $=0.90-1.10)$. In the stationary condition, the completion index grew with a time constant of $\tau=37 \mathrm{msec}$ (95\% confidence interval $=$ $22-52 \mathrm{msec}$ ) to an asymptote of $\gamma=0.76$ (95\% confidence interval $=0.66-0.86)$.

Most importantly, these results rule out the positioncue hypothesis. Relative position cues were always available, but again we obtained very different patterns of results across the three visibility conditions. Furthermore, despite large changes in the relative contrasts of occluders and targets from Experiment 1 to Experiment 2, we obtained the same general pattern of change in completion with duration in both the moving condition and the stationary condition.

\section{GENERAL DISCUSSION}

The finding that amodal completion can affect performance in perceptual tasks, but only if given enough time, is consistent with earlier reports. Our estimates of the time required for completion $(2 \tau)$ ranged from 46 to $114 \mathrm{msec}$, and averaged to $75 \mathrm{msec}$.

Although our results clearly indicate a time course for completion, our estimate of its duration should not be taken as a fixed constant of visual processing. It is becoming increasingly clear that the time required for completion is not fixed but varies with task and stimulus.

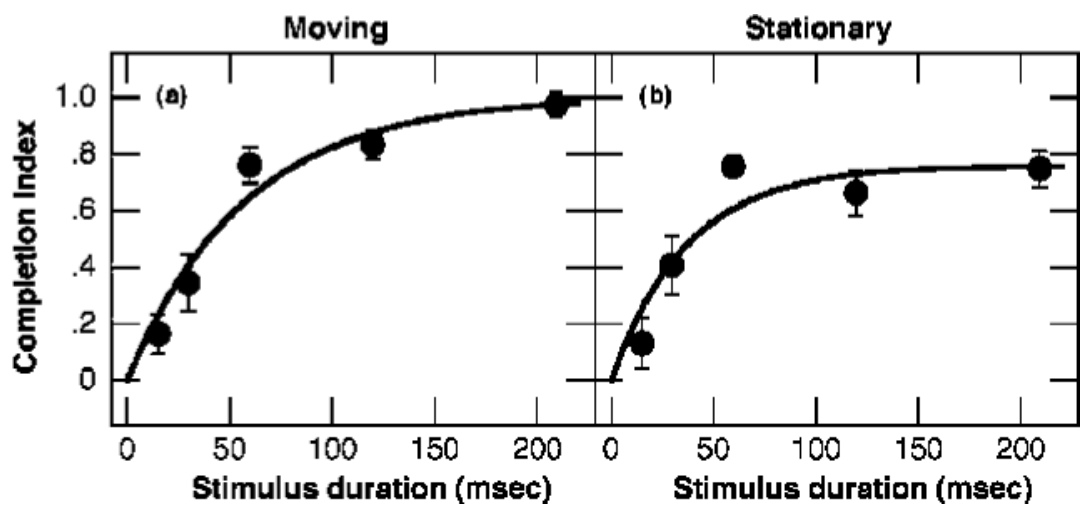

Figure 5. Results of Experiment 2. The graphs show completion indices averaged across participants. The left graph shows results from the moving condition, and the right graph shows results from the stationary condition. Error bars indicate 1 standard error. 
Sekuler and Palmer's (1992) first primed-matching study found amodal completion to require $100-200 \mathrm{msec}$; however, in a later primed-matching study, Guttman and Sekuler (2001) found that completion time varied from less than $75 \mathrm{msec}$ to over $200 \mathrm{msec}$, depending on how much of the stimulus was occluded. Shore and Enns (1997) manipulated the amount of occlusion in their stimuli, and they also found shorter completion times for smaller amounts of occlusion. Using performance in a shape discrimination task as a measure of completion, Ringach and Shapley (1996) found amodal completion to require 120-170 msec. This is longer than the estimate we found using similar methods, but their stimuli were very large $\left(17^{\circ} \times 17^{\circ}\right)$ and highly occluded $(80 \%)$, so if completion time increases with the amount of completion required, a longer time course would be expected (Guttman \& Sekuler, 2001; Shore \& Enns, 1997). Although it is difficult to compare the completion times obtained in different studies directly, the fact that studies with different methods and stimuli all conclude that amodal completion has a rapid but measurable time course provides converging evidence and makes it unlikely that the time course is an artifact of the methods employed.

Kellman and Shipley (1991) suggest that motion promotes the completion of occluded shapes by providing a cue that disconnected stimulus fragments belong to the same object. Because we tested both moving and stationary stimuli, we can address this question, which has two aspects: First, does motion speed up completion? Second, does motion increase the asymptotic strength of completion at long durations?

In Experiment 1, the difference between the completion times for the moving and stationary conditions was not statistically significant (i.e., a two-tailed $t$ test failed to reject $\left.\tau_{\text {stationary }}=\tau_{\text {moving }}, p=.30\right) .{ }^{4}$ In fact, contrary to the predicted effect, the stationary time course was faster than the moving time course; perhaps this was because all participants completed the moving conditions before the stationary conditions. In Experiment 2, there was again no significant difference between the completion times for moving and stationary conditions $(p=.11)$. We conclude that motion did not greatly speed up completion in our task.

Because amodal completion is a form of grouping, and given the importance of common fate as a grouping cue (Uttal, Spillmann, Stürzel, \& Sekuler, 2000; Wertheimer, 1923/1955), it is interesting that motion did not have a measurable effect on the speed of completion in these experiments. This result is especially surprising in light of suggestions that motion may speed up amodal completion in other situations (Shore \& Enns, 1997). It may be that other grouping and occlusion cues in our stimuli (e.g., contrast polarity, T-junctions) were so strong that motion could do little to further speed up completion. In other words, we may have experienced a ceiling effect. If so, stimuli with weaker grouping and completion cues may reveal an effect of motion on the time course of completion.

Alternatively, motion may speed up amodal completion only in some stimulus configurations. For example, Shore and Enns (1997) suggested that motion speeds up completion, but in their stimuli the unoccluded parts were spatially connected, and all moved in the same direction. In our stimuli, however, spatially disconnected object fragments moved in orthogonal directions, although the entire set of motions was consistent with the circular motion of a rigid, rectangular object. Uttal et al. (2000) showed that the strength of grouping by common fate is affected by stimulus parameters, such as interelement distance, spatial organization of elements, and direction differences among elements. Thus, the lack of an effect of motion on the speed of completion in our experiments may reflect a limiting stimulus configuration in which motion is unimportant as a completion cue. Further research is needed to determine the conditions under which motion increases the speed of amodal completion.

Turning to the question of whether motion increases the asymptotic strength of completion, we note that, in both conditions of both experiments, the completion index rose to near 1 at long durations. In Experiment 1, the difference between the asymptotic strength of completion in the moving and stationary conditions was not statistically significant (i.e., a two-tailed $t$ test failed to reject $\gamma_{\text {moving }}=\gamma_{\text {stationary }}, p=.76$ ). In Experiment 2 , the asymptote in the moving condition was significantly higher than that in the stationary condition $(p<.01)$. To be certain that these results were not artifacts resulting from a poor fit of the decaying exponential to our data, we directly compared the moving and stationary completion indices at the longest stimulus duration. This confirmed both comparisons: At $210 \mathrm{msec}$, the moving completion index was significantly higher than the stationary completion index in Experiment $2(p<.01)$, but not in Experiment $1(p=.38)$. As noted earlier, because all participants completed the moving conditions before the stationary conditions in Experiment 1, comparison of these two conditions may be confounded by practice effects. The results of Experiment 2, in which moving and stationary conditions were interleaved, are more reliable for this comparison. We tentatively conclude that motion can increase the asymptotic strength of amodal completion, and, in the following comments we suggest how to measure the effect of motion on the asymptotic strength of completion more accurately.

The main purpose of these experiments was to examine the effect of motion on the speed of completion. To do this, we required stimuli that were almost fully completed at long durations - otherwise, it would be difficult to measure the time course accurately. One consequence of this is that there was little room for differences in the strength of completion at long durations, across conditions. Our results suggest that moving stimuli were 
more fully completed at long durations than were stationary stimuli, but because almost full completion occurred even in the stationary condition (as it had to if we were to measure the time course accurately), our stimuli were not well suited for this comparison. Hence, we regard the difference in the asymptotic strength of completion as a preliminary result that should be confirmed using other stimuli that allow a greater range in the asymptotic strength of completion. Introspective studies suggest that motion does sometimes strengthen amodal completion (Kellman \& Shipley, 1991), and performance-based studies using stimuli with weaker completion cues than ours would provide a useful test of these claims.

\section{Summary}

Taken together, these experiments provide a new example of how shape discrimination can improve when a stimulus is amodally completed into a single object. This phenomenon makes it possible to measure the degree of amodal completion by comparing performance on an occluded stimulus to performance on complete and fragmented stimuli. The resulting performance-based measure reveals a time course for amodal completion, lasting around $75 \mathrm{msec}$, and shows that motion can increase the asymptotic strength of amodal completion but does not always speed up completion.

\section{REFERENCES}

BAYlis, G. C., \& Driver, J. (1993). Visual attention and objects: Evidence for hierarchical coding of location. Journal of Experimental Psychology: Human Perception \& Performance, 19, 451-470.

Behrmann, M., Zemel, R. S., \& Mozer, M. C. (1998). Object-based attention and occlusion: Evidence from normal participants and a computational model. Journal of Experimental Psychology: Human Perception \& Performance, 24, 1011-1036.

Beller, H. K. (1971). Priming: Effects of advance information on matching. Journal of Experimental Psychology, 87, 176-182.

Bruno, N., Bertamini, M., \& Domini, F. (1997). Amodal completion of partly occluded surfaces: Is there a mosaic stage? Journal of Experimental Psychology: Human Perception \& Performance, 23, 1412-1426.

DunCAN, J. (1984). Selective attention and the organization of visual information. Journal of Experimental Psychology: General, 113, 501517.

Gerbino, W., \& Salmaso, D. (1985). Un'analisi processuale del completamento amodale [A rigorous analysis of amodal completion]. Giornale Italiano di Psicologia, 12, 97-121.

Guttman, S., \& Sekuler, A. B. (2001). Visual completion: Spatial and temporal limits. Manuscript submitted for publication.

He, Z J., \& NaKayama, K. (1992). Surfaces versus features in visual search. Nature, 359, 231-233.

He, Z J., \& Nakayama, K. (1994). Perceiving textures: Beyond filtering. Vision Research, 34, 151-162.

Helmholtz, H. von (1962). Treatise on physiological optics (3rd ed.; J. P. C. Southall, Trans.). New York: Dover. (Original work published 1910)

KANIZSA, G. (1979). Organization in vision: Essays on Gestalt perception. New York: Praeger.

Kanizsa, G., \& Gerbino, W. (1982). Amodal completion: Seeing or thinking? In J. Beck (Ed.), Organization and representation in perception (pp. 167-190). Hillsdale, NJ: Erlbaum.

Kellman, P. J., \& Shipley, T. F. (1991). A theory of visual interpolation in object perception. Cognitive Psychology, 23, 141-221.
Kellman, P. J., \& Spelke, E. S. (1983). Perception of partly occluded objects in infancy. Cognitive Psychology, 15, 483-524.

Lorenceau, J., \& ShIFFrar, M. (1992). The influence of terminators on motion integration across space. Vision Research, 32, 263-273.

Meyer, G. E., \& Dougherty, T. J. (1990). Ambiguous fluidity and rigidity and diamonds that ooze. Perception, 19, 491-496.

Michotte, A., Thinès, G., Costall, A., \& Butterworth, G. (1991). Michotte's experimental phenomenology of perception. Hillsdale, NJ: Erlbaum.

Nakayama, K., Shimojo, S., \& Silverman, G. H. (1989). Stereoscopic depth: Its relation to image segmentation, grouping and the recognition of occluded objects. Perception, 18, 55-68.

Ratoosh, P. (1949). On interposition as a cue for the perception of distance. Proceedings of the National Academy of Sciences, 35, 257259.

Regan, D., \& Hamstra, S. J. (1992). Shape discrimination and the judgement of perfect symmetry: Dissociation of shape from size. $\mathrm{Vi}$ sion Research, 32, 1845-1864.

Rensink, R. A., \& Enns, J. T. (1995). Preemption effects in visual search: Evidence for low-level grouping. Psychological Review, 102, 101-130.

Rensink, R. A., \& ENNS, J. T. (1998). Early completion of occluded objects. Vision Research, 38, 2489-2505.

Ringach, D. L., \& Shapley, R. (1996). Spatial and temporal properties of illusory contours and amodal boundary completion. Vision Research, 36, 3037-3050.

Rosch, E. (1975). Cognitive representations of semantic categories. Journal of Experimental Psychology: General, 104, 192-233.

SEKULER, A. B. (1994). Local and global minima in visual completion: Effects of symmetry and orientation. Perception, 23, 529-545.

Sekuler, A. B., \& Palmer, S. E. (1992). Perception of partly occluded objects: A microgenetic analysis. Journal of Experimental Psychology: General, 121, 95-111.

Sekuler, A. B., Palmer, S. E., \& Flynn, C. (1994). Local and global processes in visual completion. Psychological Science, 5, 260-267.

SHORE, D. I., \& ENNS, J. T. (1997). Shape completion time depends on the size of the occluded region. Journal of Experimental Psychology: Human Perception \& Performance, 23, 980-998.

SNOWDEN, R. J., \& BRADDICK, O. J. (1989). The combination of motion signals over time. Vision Research, 29, 1621-1630.

Stone, L. S., Beutter, B. R., \& Lorenceau, J. (2000). Visual motion integration for perception and pursuit. Perception, 29, 771-787.

Uttal, W. R., Spillmann, L., Stürzel, F., \& Sekuler, A. B. (2000). Motion and shape in common fate. Vision Research, 40, 301-310.

Watamaniuk, S. N. (1993). Ideal observer for discrimination of the global direction of dynamic random-dot stimuli. Journal of the Optical Society of America A, 10, 16-28.

Watamaniuk, S. N., Sekuler, R, \& Williams, D. W. (1989). Direction perception in complex dynamic displays: The integration of direction information. Vision Research, 29, 47-59.

WERTHEIMER, M. (1955). Laws of organization in perceptual forms. In W. D. Ellis (Ed.), A source book of Gestalt psychology (pp. 71-88). New York: Humanities Press. (Original work published 1923)

\section{NOTES}

1. These stimuli are adapted from Lorenceau and Shiffrar's (1992) study of global motion perception.

2. One participant ran in a 75-msec condition instead of in a $105-\mathrm{msec}$ condition.

3. Because the length and width of the rectangle had to be varied across trials with high precision (accurate to less than the $0.020^{\circ}$ subtended by a single pixel), the stimuli were antialiased. Specifically, the rectangle was convolved with a rectangular pillbox function, 2 pixels wide, before being sampled by the pixel array.

4. A reviewer suggested that results in the moving condition may have been affected by a type of illusion in which a moving, occluded object appears to lose its rigidity (Meyer \& Dougherty, 1990). This would raise moving occluded thresholds relative to stationary occluded thresholds and would make it meaningless to compare the time course of the com- 
pletion index across the moving and stationary conditions. If this illusion affected our results, it would presumably have been strong at long stimulus durations, where the motion of the occluded square was most noticeable. In fact, the asymptote of the completion index in the moving condition was not significantly less than 1 , indicating that the participants were as good at judging the aspect ratio of the stimulus in the moving occluded condition as they were in the moving complete condition. Furthermore, the asymptote in the moving condition was higher than the asymptote in the stationary condition in both experiments (though significantly higher only in Experiment 2). We conclude that this type of illusion did not have an appreciable effect on thresholds in the moving occluded condition.

(Manuscript received April 19, 2000; revision accepted for publication April 11, 2001.) 